\title{
Bias in the ELO-System of Online Chess
}

\begin{abstract}
ELO is the key performance indicator in chess, a global and worldwide measure of chess skills and strength of chess play. Since their invention, ELO-systems are intended to be comparable and unbiased, so that chess players can know their level of play and can compare it among systems and internationally. Moreover, ELO is the defining feature of grandmasters with legal implications. Thus, it is highly important to only have unbiased ELO-system, whenever a terminology bearing ELO is used. However, most recent online chess sites seem to utilize a highly biased ELO-system that can maybe not be compared to tournament or FIDE chess ELO-score systems. It could be that online ELO-systems are strategically biased to make all hobby players around the world appear less professional by systemically down-shifting the ELO-score system. This short report offers some insights about this potential ELO-deflation phenomenon in online chess. It can be shown that online ELO-systems do not reflect real chess skill strength in ELO as measured by different methods: (1) game strength does not reflect tactical or strategic strength, (2) there is a shift in ELO-distribution between hundreds and up to thousand ELO-points of the average level player, e.g. FIDE RBB versus an online site, and (3) Elometer, a scientifically approved and scientifically standardized method also reveals this dramatic discrepancy and ELO-reduction. The reason for this deprivation of ELO-points seems to be an artificial ELO-scarcity that is introduced into the system whenever a new player enters the site and has to start with fewer ELOpoints than his or her skill level. There are not enough aggregated ELO-points that could mirror the sum of all chess skills in online chess and year after years it seems to worsen, while FIDE-ratings are rising slightly. Hence, this work reminds that the total ELO-points per rating system must be always adjusted, and there could be a strategic bias to downgrade all chess players. Millions of chess-players world-wide might be extremely underrated and their chess skills seem to be wrongly assessed and viewed.
\end{abstract}

Keywords: False, bias, ELO, Glicko, ranking, chess, online, play, game, gaming, UCFS
Volume I Issue 2 - 2018

\section{Roman Anton}

The University of Truth and Common Sense, Department of Theoretical Sciences, Germany

Correspondence: Roman Anton, The University of Truth and Common Sense, Department of Theoretical Sciences, Germany, Email mail.roman.anton@gmail.com

Received: January 08, 2018 | Published: March 08, 2018

\section{Introduction}

There are millions of chess players around the world that play chess on websites like chess.com. ${ }^{1}$ According to chess.com, ${ }^{1}$ there are 600 Million chess-players worldwide, and more than 20 Million members on chess.com ${ }^{1}$ that play up to 1 Million games per day, and there are 360.000 tournament players and 1594 grandmasters of which only $2.2 \%$ are female, while it is free to play for everyone. ${ }^{1}$ Today, bias in research is ubiquitous, ${ }^{2}$ also in chess research and most likely also in ELO rating, which should be further analyzed in some more details. The strength of a player in the game of chess and additional zero-sum games is usually estimated and assessed in ELO, a rational chess skill rating system that was initially developed by Arpad Elo, a Hungarian physicist who lived, worked and taught in the USA. The ELO rating system statistically derives numerical outcomes in ELO of the games a player plays against opponents of different strength: it increases the ELO score value of the winner as much as it decreases the ELO score of the loser, roughly speaking, deviations of the rule equal much out as the sum of both directions. A high-rated player can lose more points against a low-rated player, who can win more points against a high-rated player, and vice versa, a high-rated player can win fewer points against a low-rated player, who will lose fewer points against a high-rated one, leading to an ELO conservation effect after deviations and correction factors equal much out, slightly comparable to the conservation of energy that is fixed in the system which is only partially corrected. This adjustment upwards or downwards goes back to $\mathrm{Elo}^{3,4}$ who suggested it, and USCF, the United States Chess Federation, implemented his suggestion in 1960, subsequently it became an international standard, and USCF, the United States Chess Federation, has implemented his suggestions in 1960. The basis of ELO's formula ${ }^{3}$ for the logical probability function expresses the expected score that is always further scalable for ELO changes per game. It is represented by the expected score (ExScore) of player A and B:

ExScore $_{A}=\frac{1}{1+10^{E L O(B)-E L O(A) / 400}} \quad ;$ ExScore $_{B}=\frac{1}{1+10^{E L O(A)-E L O(B) / 400}}$

Hence, an ELO difference of 400 results in a 10-times scaled expected score and is part of the ELO-change calculation ${ }^{3,4}$ : if a specific player is expected to win in $40 \%$ of all points in games against a stronger player and wins $50 \%$ of them, then the ELO would rise but would stay the same at $40 \%$ of wins, in general. The scale of the scoring is given by a factor $\mathrm{x}$, which modulates the amount of ELO-point-changes and which generally does not much alter the overall aggregated ELO points in the system. There have been some advancements but mainly in the fine-tuning of the ELO system, such as the Glicko or Glicko-2 ELO systems ${ }^{5}$ that is used on online chess-sites, like chess.com, ${ }^{1}$ or by the Australian Chess Federation, and further online chess sites. As a suitable improvement of factor $\mathrm{x}$, Glicko-1/2 uses the statistical rating deviation and volatility to integrate the error from the ELOvalues of the two players. As a result, Glicko systems faster adjust the ELO rating acurately but only up to the level of the specific rating system and the historical preset ranking, which can be either correct or not, right or wrong, correct or giving a too high or too low ELO scoring. Consequentially, the mean ELO will be falling, as any upward-adjustment costs the system ELO twice. Over time, a correct 
adjustment would become impossible for probably $99 \%$ of players.

\section{Results and discussion}

Millions of professional and hobby chess players are playing and learning chess on online chess gaming sites. Online chess sites like chess.com ${ }^{1}$ play also a big role on chess of today, the chess community, the chess culture, but also chess learning and training and more than 100 Million players have used and played on an online chess site. These sites provide great benefits for chess players as they concentrate information, tools, news, articles, statistics, games, analysis, tools, opening books, lessons, ideas, puzzles, and much more to chess players. They represent an ideal place to quickly find an opponent of your strength and length of the game you want to play. Since a huge proportion of chess-players are using, mainly or only, chess websites to play chess, these sites are becoming a commons infrastructure and platform of chess, like any other major websites including search engines. They are already today a commons for which all users get a right of correct treatment, such as the ELO-comparability, which can be seen as a qualification like a certification or an official testing of a skill, which of course must be unbiased and correct. No matter if Glicko-1, Glicko-2, or UCFS, all ELO rating systems must be always comparable to one ELO scale per game type, e.g., rapid or blitz, achievable by using a simple ELO standardization. It is a transition from a private function to a public platform and infrastructure that many websites are going through today: whenever a societal function is in play, the user rights must be better protected. Here, it is very important that onlinechess sites have a functioning, reliable and comparable ELO-system in place, as the success in chess for most players is found in ELOvalues, especially for the majority of players that visits these sites. The initial and longstanding idea of Apard Elo's chess rating is a better comparability of chess players strength, which Dr. Elo had achieved, ${ }^{4}$ while Glicko or Glicko-2 can be seen as a statistical refinement, a fine-tuning that makes use of given rating deviations and players to achieve a faster real ELO-adjustment with less volatility and less games are needed - but it does thereby only partially correct the overall ELO-points in the system, which still leads to a ELO-deflation over time if average skill strength is higher than average ELO-starting value and vice versa. Initially it was configured to let players start with 1500 ELO-points and a rating deviation of 350, which is 300 ELO-points higher than on chess.com, ${ }^{1}$ where everybody has to start with only 1200 ELO points. Thus, the chess-sites broke the most basic standardization of a 1500-ELO starting value. The average skills of active players is believed to be even higher and not lower, one must assume, as an ELO of 1600 on a scale of 3000 already represents a player that can beat 999 of 1000 chess players worldwide, as the top 200.000 and top $0.1 \%$ of all players. Glicko is a rational and suitable amendment to the ELOsystem which initially only used a manually adjustable a factor $\mathrm{x}$ for scaling that could differ between top players and amateur tournaments or matches. Glicko ELO rating uses the rating deviation instead to assure a higher certainty and a lower volatility in a faster way. But during this adjustment a too low rated player will still reduced the aggregated ELO points in the rating sytem twice. As Glicko can only partially adjust it and is not build to cope with a strongly introduced systemic bias and can only milden it.

As a result, these novelties further improved the speed of accuracy in ELO in fewer games and the deviation of ELO rating, which both makes the ELO-system more accurate and comparable, but only works fine if everything else is done right, i.e. correct starting values. However, since $10-20$ years, many players might have noticed it, it has become seemingly impossible to obtain a suitable and comparable ELO rating on one of these chess-sites in the range of 1000-2000 ELO. The question arises how such a widely "perceived bias" can or could happen in such sophisticated and statistically elaborated ELO or the Glicko-1/2 rating system that should bear the quality of , as Glicko stems from Harvard. It is also clear that any ELO rating only makes sense if it is comparable among all platforms and players. It is the idea of any chess rating system that it must be comparable. You cannot simply say we are using Glicko and thus one cannot compare them with another rating system - they all should be comparable, Glicko is just the refinement of ELO, only a fine-tuning if you will - that forgot about the impact of the ELO-starting-value problem. So, these empirical questions arise: are they really not comparable, what is the level of deviation to real ELO-values, and how can you make them comparable again in between rating systems and also worldwide?

\section{Amount of Players per ELO Segment}

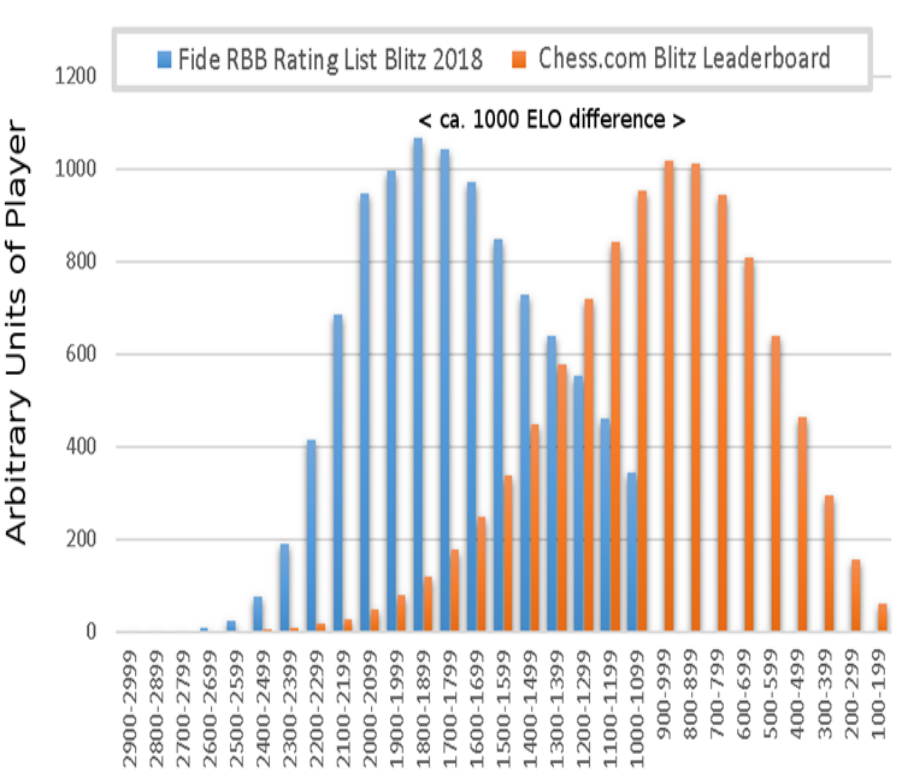

Figure I Comparison of two statistical distributions of rated players in blitz games at chess.com (blue) and FIDE (orange). Please notice the shift of 1000 ELO points between the peaks of the two chess rating systems.

The answer to these questions could be given by the following hypothesis about the rating: Whenever a new player enters a site like chess.com ${ }^{1}$ or ChessBase, Chess Cube, ICC, he or she will start with a number of ELO points and a rating deviation in the case of Glicko-1 or Glicko-2.,5 These sites have always argued that this would not be a problem, as over time the player will receive right ELO values, and Glicko would even accelerate this by using more suitable rating deviations. But like in the mathematical field of differential equations, the initial value problem arises. The 'initial condition' would be the starting ELO value and its rating deviation in a system of entities that have different strength and likelihoods to win, draw and lose. As a result, the amount of ELO-points will stay at a specific aggregated level in the system, which can be too low for all players. If we game-theoretically assume, there are the three following players in a hypothetical tournament: one player with a real skill strength (RSS) of 1000 RSS-ELO, one with 2000 RSS-ELO, and also one with 3000 RSS-ELO. Hypothetically and very simplified, if these players now all would start with 1200 ELO points each - as on chess. $\operatorname{com}^{1}$ - and would 
now play many games, it would result in a fair rating only for the 3000 RSS-ELO player, who could theoretically even maybe achieve 2900 ELO on the online chess website, as nobody has the RSS to deprive his ELO points. But the player with an RSS of 2000 ELO could only achieve 1600 of the remaining ELO points in the system, and the player with 1000 ELO could theoretically even receive 0 ELO points this way on this website for the three player, as as the remaining two players can maybe fully deprive all points. Glicko could only slightly improve this. This hypothetical example game illustrates what kind of issue the ELO-system generates that maintains a too constant amount of ELO points per system. Grandmasters at a level of 3000 might be still a tiny minority, and there are worldwide only 4-5 players with a real rating of 2900-3000 ELO points. ${ }^{6}$ The majority of players will still have an RSS-ELO between 1000 and 2000 ELO-points, which is the part of the statistical distribution that would be the most erroneous and false rating due to the artificial scarcity of overall ELO-points in the entire system - caused by the Cauchy problem, the too low 'initial value' of 1200 ELO points or 1300 , or 1400 , depending on the online chess website.

To test this hypothesis, the number of players in blitz games were compared between official RBB FIDE ratings and blitz games on chess. $\operatorname{com}^{1}$ by plotting the relative amount of players per 100-ELO-segment (Figure 1). In fact, an unexpected gigantic shift in the curve and peak can be, observed (Figure 1). Almost 1000 ELO points subdivide the peaks of both ELO rating systems (Figure 1). Hence, this further indicates that comparability seems most likely not to be given between such FIDE-ELO and chess.com ${ }^{1}$ ELO rating systems. A major deflation of ELO points must be assumed on online chess websites, as all of them have too low initial ELO values.

To solve this problem, every chess ELO rating system should advise a roughly correct amount of ELO points to every new player already from the beginning: which means that a 1000 RSS-ELO should achieve 1000 ELO over time, a 2000 RSS-ELO player should receive 2000 ELO points and a 3000 RSS-ELO player must still get 3000 ELO. The challenge is to identify the ELO reflecting the real skill strength (RSS) of the individual player. If the initial value, the starting ELO, would be correct, the problem would be much smaller as the overall amount of points to compete for is more correct. But the players would still get better over time, one might assume, and this would not increase the overall amount of ELO points in the system (see Supplementary Figure 1and Figure 2) - it is a monetary-type ELO problem. Centrally, one should not cause an ELO inflation nor a deflation; one should assure stability and comparability so that ELO values are correct for all players, which still drift over time as players come and go, get worse or better, also on average and in sum. To do this, the overall aggregated ELO-points must reflect the overall RSSELO of all the players to make the ELO/Glicko system work.

One may not assume without any reason that the ELO of all dedicated online players is below the absolute amateur level of 1000 points (see Figure 1), which would approximate a beginners stage of learning the major rules of chess. Most players know the openings and have much higher tactical skills and already play very competitive on very average ELO levels. Experience also tells, that two decades ago ELO indicated a totally different skill strength of a player. There are too many good players in the world to assume this. People that visit chess. com $^{1}$ are chess fans and people interested in chess, they are really not so bad players one might surely assume, also in light of up to 600 million chess players worldwide. ${ }^{1}$ If only $0,33 \%$ of players reach a level of 1600 ELO there must be something gone wrong causing this that has caused this strong ELO-deflation.

One way to technically test and repair the ELO scoring is shown by Elometer that uses item response theory ${ }^{7,8}$ to derive an ELO maximumlikelihood estimate using a set of chess problems of the Amsterdam Chess Test $^{9}$ with known properties, based on a prediction formula regressing the RSS of 259 players, all participants of the Dutch Open Tournament, ranked from 1169 to 2629 on the Birnbaum model. ${ }^{7,10}$

Elometer ${ }^{7}$ thereby tries to reveal the real ELO-strength of a player using the above mention methods. By using Elometer, ${ }^{7}$ the hypothesis that the ELO-system is erroneously biased, and not comparable, could be again further substantiated (Figure 2): A typical chess.com ${ }^{1}$ player with a skill strength of 1900 ELO points in puzzles, 2000 in lessons/ position/strategy, achieved an ELO of 2015 at Elometer (95 CI, 1891 - 2140), which is shown by evidence in Figure 2 and compared to the chess.com ${ }^{1}$ ELO rating (Figure 3), which was much lower at only 1475 ELO-indicating a potential deflation of about 500 ELO-points in online chess. Hence, two things can be derived: (i) there is no ELOrating comparability but an urgent requirement of it, and (ii) Elometer ${ }^{7}$ provides a key to find a first and robust solution: by assessing the RSSELO that could be theoretically used used to correct the initial values, as the ELO-deflation mainly stems from stems from false starting values and drift.

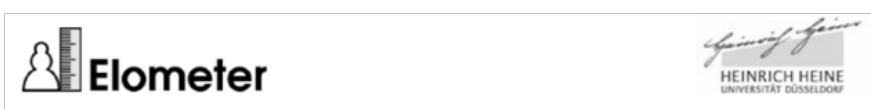

\section{Thank you for participating!}

Based on your move choices, our estimate of your Elo rating is 2015, with a $95 \%$ confidence interval of
[1891...2140].

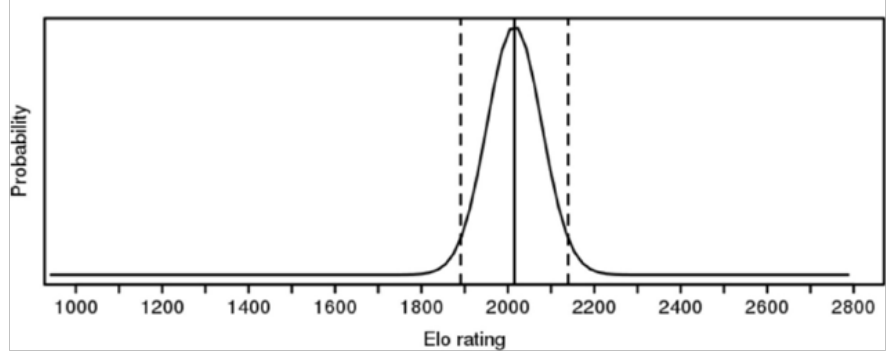

Figure 2 Scientific ELO estimation based on Elometer's item response theory (January 2018).A 95\% confidence interval (dashed lines) is shown for the estimate (solid line). Please notice, this tournament-related scientific estimate of 2015 ELO corresponds to only I475 ELO at chess.com in this example.

If all new chess players would do a testing like the scientific Elometer assay ${ }^{7}$ this ELO-number could be used to improve the initial rating problem that causes the deflation, of all players that enter the gaming system. The learning curve of all players could be also assessed in this way to adequately increase the overall amount of ELO-points (see Supplementary Figure $1+2$ ). Consequentially, the total amount of ELO-points in the system would be more accurate and the slope and steepness of the curve would not be so much distorted as it might be today (Figure 1). One could assume, that there are only a few players that start at the right ELO, mainly grandmasters that enter the site and who may start somewhere around or at their known ELO value, but the majority of players are not grandmasters and 20 Million false initial valued ELO-scores could, in fact, dramatically shift the entire ELO-curve and would make it extremely steep - as can be maybe seen in Figure 1 in a problem-revealing way. 


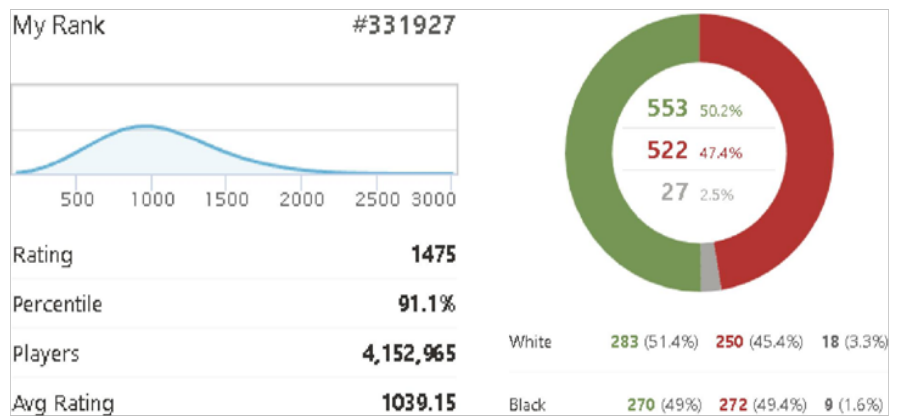

Figure 3: Please notice, this tournament-related scientific estimate of 2015 ELO corresponds to only I475 ELO at chess.com in a representative example and it is likely to be true for most chess players. An ELO of I475 recently relates to the upper $10 \%$ (percentile $91.1 \%$ ) in January 2018; this seems to be very biased and atypical for real-world chess and a curiosity of online chess mainly. This bias could be easily fixed via better starting values to assure the right level of aggregated ELO points in the rating system.

Another way to solve this is to always release enough ELO-points into the competitive situation on the chess site. Simply calculate what one has to expect in a normal distribution of chess hobby players and professionals, take the overall points, and fill up the online chess game site with additional ELO-points up to the level of the aggregated sum of all of these ELO-values, so that enough points are in the system that represents a normal distribution of all all, which will only minimally change over time due to the vast amount of players. But how to best assess the normal distribution of all players RSS-ELO? Estimates can be simply based on (A) historic tables or (B) RSS-ELO, or (C) a new formula. ${ }^{7,9}$

\section{Supplementary Material is available using this hyperlink.}

\section{Conclusion}

This short report concludes that an ELO-deflation is likely to be found in most online chess rating-systems as most, if not all, use too low initial ELO-values. Consequentially, all online ELO rating systems should be checked and made comparable for all players, whenever a deflation (or inflation) can be found. The first ELOsystems circumvented this problem by giving adequate initial ELO values, this is also done in chess clubs around the world. Reasonably, this should be also done in online chess to avoid an ELO-deflation of a bigger type, as ELO must be comparable at any given level of ELO and play. The individual ELO score can only be correct if the overall aggregated ELO-points in the system are correct too. This can only be achieved with right initial ELO-scores and by introducing new freely floating ELO-points into the entire system up to the level of the expected aggregated normal distribution, and subsequently letting all of the players compete for them in a in a fair way (see Formula 1 in the supplementary material). Once the system works, all additional driftadjustments can be done with the initial ELO values. This should be done without any artificial ELO-scarcity that could cause a steepness of the ELO-distribution especially between 100-2100 ELO, what could be maybe called an ELO-deflation shift whenever it is verified. The magnitude of the deflation seems to vary from ELO-level to ELOlevel and one can only speculate about how many hundreds of ELOpoints are deviating from a player's RSS-ELO. An ELO-gold standard is missing and should be included for all levels of chess players to prevent any deflation that can happen over time. Glicko only reduces the deflation but does not fully prevent it as it benchmarks with the aggregated points and very slightly but still significantly reduces them by every new player. Hence, an optimization of Glicko is here suggested: (a) correct initial ELO starting values and (b) any points lost against a new player must be added back to the system until his natural ELO is reached, (c) learning curve effects of the pool of players should be included in times of improved learning (see Supplementary Figure 1 and Formula 1), (d) overall aggregated ELO-points must reflect overall aggregated RSS-ELO points. ELO biases seems not to be an issue for the grandmasters on the site as they enter the site with their own points and they are maintained in a top players bubble where they might mainly play with other grandmaster-level players. There is not so very much contact with the average chess player's world where you face harsh ELO-slopes and systemic ELO scarcity, as there are only very few players better than them that would be able to deprive them of the artificially scarce ELO points. As a result, mainly the stronger players could cause this ELO-deflation shift for weaker players, or the entire curve could be shifted also. Generally speaking, ELO-inflation and ELO-deflation must be better avoided in online chess and this requires active measures that refill the pool of aggregated ELO-points up to the level of the recent ELO-RSS of all players. ELO-gold-standards and ELO-tests like the Elometer assay could be utilized to adjust the initial ELO-values, or to better normalize the ELO curve or distribution. The intrinsic trend towards artificial scarcity of ELO-points and ELO-score aggravation steepening from 100-2000 ELO points must be ended and avoided by via suitable normalizations and enough total ELO points must be in the system to ease the steepening ELO slope. Millions of frustrated chess players might want to have a more acurate, a more reliable and amore comparable ELO rating - and millions of player might have been systemically underrated since decades. ELO is a certification like IQ or an exam grade that must meet the claim to be correct. Millions of players might have been damaged in sum by this systematic ELO-downgrade for the majority of players: for instance, on the job market, in the eyes of others, and even in their own eyes. Most players could be much better than previously anticipated. This should be tested further and steadily for all online chess websites to keep all ELO rating systems comparable and more correct. Maybe the life of many million chess players are improved if they receive the correct ELO values that they strive for since some time.

\section{Acknowledgements}

University of Truth and Common Sense is acknowledged here.

\section{Conflicts of interest}

The authors declared that there are no conflicts of interest.

\section{References}

1. Chess.com. Chess.com [Internet]. 2018.

2. Rothstein H, Sutton AJ, Borenstein M. Publication Bias in Meta Analysis Prevention, Assessment, and Adjustments. Sutton and Borenstein, editor. Chichester, England: Wiley. 2005;1-376.

3. Elo A. 8.4 Logistic Probability as a Rating Basis". The Rating of Chessplayers, Past\&Present. Bronx, NY: ISHI Press International.

4. Elo A. The Rating of Chess Players, Past and Present. Arco.; 1978.

5. Glickman ME. The Glicko system. 2016.

6. FIDE. FIDE Rating Statistics.2018.

7. Diedenhofen B, Musch J. Elometer [Internet]. The Heinrich Heine University of Düsseldorf. 2018. 
8. Hambleton R, Swaminathan H, Rogers H. Fundamentals of item response theory. Sage. Newbury CA. 1991.

9. Maas H, Wagenmakers EJ. A psychometric analysis of chess expertise. Am JPsychol. 2005;118(1):29-60.
10. Lord FM. Applications of item response theory to practical testing problems. Mahwah, NJ: Lawrence Erlbaum; 1980. 